\title{
Effect of Platelet Activating Factor-Acetylhydrolase on the Formation and Action of Minimally Oxidized Low Density Lipoprotein
}

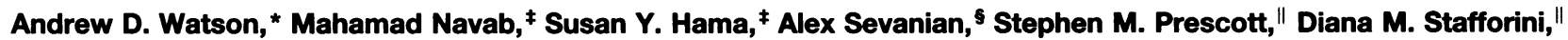 \\ Thomas M. Mclntyre," Bert N. La Du, ' Alan M. Fogelman, ${ }^{\ddagger}$ and Judith A. Berliner ${ }^{\star \ddagger}$ \\ Departments of $*$ Pathology and ${ }^{\ddagger}$ Medicine, University of California, Los Angeles, California $90024 ;{ }^{\S}$ Institute for Toxicology, University \\ of Southern California, Los Angeles, California 90007; "Department of Medicine, University of Utah, Salt Lake City, Utah 84112; and \\ the 'Department of Pharmacology, University of Michigan, Ann Arbor, Michigan 48109
}

\begin{abstract}
Mildly oxidized low density lipoprotein (MM-LDL) produced by oxidative enzymes or cocultures of human artery wall cells induces endothelial cells to produce monocyte chemotactic protein-1 and to bind monocytes. HDL prevents the formation of MM-LDL by cocultures of artery wall cells. Using albumin treatment and HPLC we have isolated and partially characterized bioactive oxidized phospholipids in MM-LDL. Platelet activating factor-acetylhydrolase (PAFAH), a serine esterase, hydrolyzes short chain acyl groups esterified to the $s n-2$ position of phospholipids such as PAF and particular oxidatively fragmented phospholipids. Treatment of MM-LDL with PAF-AH $\left(2-4 \times 10^{-2} \mathrm{U} / \mathrm{ml}\right)$ eliminated the ability of MM-LDL to induce endothelial cells to bind monocytes. When HDL protected against the formation of MM-LDL by cocultures, lysophosphatidylcholine was detected in HDL; whereas when HDL was pretreated with diisopropyl fluorophosphate, HDL was no longer protective and lysophosphatidylcholine was undetectable. HPLC analysis also revealed that the active oxidized phospholipid species in MM-LDL had been destroyed after PAFAH treatment. In addition, treatment of MM-LDL with albumin removed polar phospholipids that, when reisolated, induced monocyte binding to endothelial cells. These polar phospholipids, when treated with PAF-AH, lost biological activity and were no longer detected by HPLC. These results suggest that PAF-AH in HDL protects against the production and activity of MM-LDL by facilitating hydrolysis of active oxidized phospholipids to lysolipids, thereby destroying the biologically active lipids in MM-LDL. (J. Clin. Invest. 1995. 95:774-782) Key words: LDL modification - lipid peroxidation $\cdot$ albumin $\cdot$ monocyte adhesion $\cdot$ platelet activating factor-acetylhydrolase
\end{abstract}

\section{Introduction}

Oxidation of LDL appears to play an important role in the initiation and promotion of atherosclerosis $(1-3)$. The role of

Address correspondence to Andrew D. Watson, Department of Pathology, Center for Health Sciences, Room 13-186, UCLA Medical Center Los Angeles, CA 90024-1732. Phone: 310-825-2436; FAX: 310-2065178.

Received for publication 18 October 1993 and in revised form 9 September 1994.

J. Clin. Invest.

(c) The American Society for Clinical Investigation, Inc.

0021-9738/95/02/0774/09 \$2.00

Volume 95, February 1995, 774-782 lipid peroxidation in oxidative modification of LDL has recently been reviewed (4). The extent to which LDL is oxidized has a fundamental influence on its physical and biological properties in vitro. The events that occur during the oxidation of LDL and the composition of the final products probably depend on a variety of factors, some of which may be the oxidative initiator; the antioxidant content and lipid composition of LDL; the transition metal content and $\mathrm{pH}$ of the buffer; and the method and techniques by which the LDL was isolated. In the literature, " oxidized LDL" probably represents a broad spectrum of modified particles ranging in degree of oxidation from mild to extensive. Highly oxidized LDL (Ox-LDL) ${ }^{1}$ contains abundant lipid peroxidation products (5-7); has increased electrophoretic mobility $(8,9)$; is taken up by the scavenger receptor $(10,11)$; and is cytotoxic to vascular cells (12-14). In contrast, mildly oxidized LDL (MM-LDL) contains fewer lipid peroxidation products; is taken up by the native LDL receptor as shown by competition studies with native LDL (15); induces endothelial cells to bind monocytes $(15,16)$; and stimulates the production of monocyte chemotactic (17) and differentiation factors (18). It has previously been shown that cocultures of human aortic endothelial and smooth muscle cells convert LDL to MM-LDL, which can subsequently induce endothelial cells to produce a factor that causes monocyte chemotaxis and transmigration into the subendothelial space (19). This transmigration is completely blocked by antibody to monocyte chemotactic protein1 (19).

In humans, plasma levels of HDL are inversely correlated with risk of clinically significant coronary events $(20,21)$. Moreover, in vitro experiments have demonstrated that HDL inhibits LDL oxidation $(14,22)$, Ox-LDL-induced inhibition of endothelium-dependent relaxation (23), and modification of LDL to MM-LDL (19). Elucidation of the mechanism(s) by which HDL prevents LDL modification may provide clues to its beneficial effects in vivo. We have previously shown (15) that the activity of MM-LDL partitions with the polar lipid fraction and here we show that particular phospholipids can be isolated from MM-LDL by transfer to albumin and HPLC. These transferred phospholipids were found to induce endothelial-leukocyte interactions identical to those induced by MM-

1. Abbreviations used in this paper: BHT, butylated hydroxyltoluene; DFP, diisopropyl fluorophosphate; HAEC, human aortic endothelial cells; HASMC, human aortic smooth muscle cells; LPC, lysophosphatidylcholine; MM-LDL, mildly oxidized LDL; MTM, monocyte transmigration; Ox-LDL, highly oxidized LDL; PAF-AH, platelet activating factor-acetylhydrolase; PAPC, 1-palmitoyl-2-arachidonoyl-sn-glycero3-phosphorylcholine; PC, phosphatidylcholine; PUFA, polyunsaturated fatty acids. 
LDL. We have hypothesized that HDL may inhibit the formation of MM-LDL by modulating the production and/or stability of biologically active phospholipids in MM-LDL. We have focused on the effect of platelet activating factor-acetylhydrolase (PAF-AH) which protects against $\mathrm{Cu}^{2+}$-induced oxidative modification of LDL (24). PAF-AH plays an integral role in the regulation of inflammatory responses by hydrolyzing the acetyl moiety located in the $s n-2$ position of PAF, a potent vasoactive phospholipid $(25,26)$. PAF-AH can also hydrolyze phospholipids that possess short chain acyl groups in the $s n-2$ position resulting from oxidation and subsequent fragmentation of the native esterified polyunsaturated fatty acids (PUFA) resulting in the formation of lysophosphatidylcholine (LPC) $(27,28)$.

In this study we have isolated and partially characterized biologically active lipids in MM-LDL and have shown that HDL-associated PAF-AH can alter these lipids and abolish their biologic activity. We, therefore, suggest that PAF-AH may play an important role in the initiation of atherogenesis by modulating the production and biological activity of MM-LDL.

\section{Methods}

Materials. Tissue culture media, serum, and supplements were obtained from sources previously reported $(15,19)$. Acetonitrile, chloroform, hexane, methanol, 2-propanol, triethylamine (all HPLC grade or better) were obtained from Fisher Scientific Co., Pittsburgh, PA. Diisopropyl fluorophosphate (DFP), gelatin (endotoxin-free, tissue culture grade, No. G 9391), butylated hydroxytoluene (BHT), and BSA (essentially fatty acid free, low endotoxin) were obtained from Sigma Chemical Co., St. Louis, MO. Authentic 1-palmitoyl-2-arachidonoyl-sn-glycero3-phosphorylcholine (PAPC) and 1-palmitoyl-2-linoleoyl-sn-glycero-3phosphorylcholine, were obtained from Avanti Polar Lipids, Alabaster, AL. TLC silica plates and HPLC analytical and guard columns were obtained from Alltech Associates, Inc., Deerfield, IL. Aminopropyl and octadecyl solid phase extraction columns were obtained from J. T. Baker Inc., Phillipsburg, NJ. Transwells and chamber slides were obtained from Costar Corp., Cambridge, MA. The fluorescent probe 1,1'-dioctadecyl-3,3,3',3' tetramethyl-indocarbocyanine perchlorate (DiI) was purchased from Molecular Probes Inc., Eugene, OR. Centricons were obtained from Amicon Corp., Beverly, MA. PAF-AH was isolated from human erythrocytes as described (29).

Endothelial cell cultures. Rabbit aortic endothelial cells (RAEC) at passages 9-17 and human aortic endothelial cells (HAEC) at passages 4-7 were cultured as described $(15,19)$. Porcine aortic endothelial cells were isolated by enzymatic digestion according to the method of Rosenthal et al. (30) with modifications. Porcine aortic strips were incubated in a $0.2 \%$ solution of collagenase in medium 199 and $1 \times$ PBS (1:1) for $10 \mathrm{~min}$ at $37^{\circ} \mathrm{C}$. The endothelial cells were dislodged by a stream of medium $199+5 \%$ heat-inactivated FBS containing $1 \%$ penicillin-streptomycin-glutamine and $0.05 \%$ fungizone. Cells were seeded in wells coated with $0.1 \%$ gelatin then grown and propagated in medium $199+10 \%$ heat-inactivated FBS containing $1 \%$ penicillinstreptomycin-glutamine and $0.05 \%$ fungizone.

Cocultures. HAEC and human aortic smooth muscle cells (HASMC) were isolated as previously described (19). Transwells or chamber slides were used for formation of cocultures and for the study of monocyte transmigration. The wells were treated with $0.1-0.5 \%$ gelatin at $37^{\circ} \mathrm{C}$ overnight. HASMC were seeded in units or on membranes at a confluent density of $1 \times 10^{5}$ cells $/ \mathrm{cm}^{2}$ and were cultured for $3 \mathrm{~d}$ at which time they had covered the entire surface of the well and had produced a substantial amount of extracellular matrix. The HASMC surface was treated with human fibronectin, $20 \mu \mathrm{g} / \mathrm{ml}$ medium 199 for $10 \mathrm{~min}$, which was removed before seeding with HAEC. HAEC were subsequently seeded at $2 \times 10^{5}$ cells $/ \mathrm{cm}^{2}$ and were allowed to grow, forming a complete monolayer of confluent endothelial cells in $2 \mathrm{~d}$. In all experiments, HAEC and autologous HASMC were used at passage levels of three to six. Identical results were obtained with multilayer cocultures produced in the different systems (i.e., transwells or chamber slides). Blood monocytes were obtained from a large pool of healthy donors by a modification of the Recalde procedure (31).

Lipoproteins. LDLs $(d=1.019-1.069 \mathrm{~g} / \mathrm{ml})$ and HDLs $(d$ $=1.069-1.210 \mathrm{~g} / \mathrm{ml}$ ) were isolated from the sera of normal blood donors by density gradient ultracentrifugation as described (32) and were used within 1-2 wk of isolation. In later stages of these studies, LDL isolation was accelerated by the use of $100,000 \mathrm{~mol}$ wt cut off Centricons (Amicon Corp.) for desalting, and was completed in $48 \mathrm{~h}$. The concentration of lipoproteins is expressed in terms of protein content throughout this report.

Lipoprotein modification. MM-LDL was produced enzymatically by the addition of soybean lipoxygenase bound to $\mathrm{CNBr}$-activated beads along with linoleic acid ( $187 \mathrm{mM}$ ) to LDL in PBS for $24 \mathrm{~h}$ at $37^{\circ} \mathrm{C}$. Oxidation was terminated by the addition of EDTA and BHT at 100 $\mu \mathrm{M}$ and $0.3 \mathrm{mM}$, respectively. MM-LDL produced by this method has significantly less LPC than MM-LDL produced by our previous enzymatic method (33). We have obtained active preparations of MMLDL by prolonged storage at $4^{\circ} \mathrm{C}$, mild oxidation with iron, and incubation with cocultures which contain low levels of LPC. We thought it was important to minimize the LPC content in MM-LDL since others have reported effects of LPC on endothelial cells in vitro. The concentration of bacterial endotoxin in medium containing each agonist was $<50$ $\mathrm{pg} / \mathrm{ml}$ (determined by chromogenic assay) which is $\sim 50$-fold less than that required to induce monocyte binding.

Lipid extraction. Lipids were extracted using a modification of the method of Bligh and Dyer (34). To 1 vol of aqueous lipid-containing solution $7 \mathrm{vol}$ of chloroform/methanol (2:1) containing $0.01 \%$ BHT was added. The mixture was vortexed at high speed for 1-2 min and then centrifuged at $1,200 \mathrm{~g}$ for $15 \mathrm{~min}$ at $4^{\circ} \mathrm{C}$ (model J-6B; Beckman Instruments, Inc., Fullerton, CA) equipped with a JS-4.2 rotor (Beckman Instruments, Inc.). The chloroform phase was carefully removed and 5 vol of chloroform was added to the residual aqueous phase and protein interface. The mixture was vortexed and centrifuged as above and the chloroform phase was pooled with the previous extract. Phospholipid recovery typically ranged between 96 and $98 \%$ as determined by spiking LDL with known quantities of 1-palmitoyl-2[ $\left[{ }^{13} \mathrm{C}\right]$ arachidonoyl-sn-glycero-3-phosphorylcholine and 1-palmitoyl- $2\left[{ }^{3} \mathrm{H}\right]$ linoleoyl-sn-glycero-3phosphorylcholine.

Solid phase extraction chromatography. Phospholipids, free fatty acids, and neutral lipids (cholesterol, cholesteryl esters, mono- di- triacylglycerides) were separated essentially by the method of Kaluzny et al. (35) utilizing aminopropyl solid phase extraction chromatography. Total lipid extracts were dried to a lipid residue under nitrogen at $37^{\circ} \mathrm{C}$ and resuspended in $\sim 250 \mu \mathrm{l}$ of chloroform. The extracts were then added to aminopropyl spe columns that had previously been washed with $3 \mathrm{ml}$ methanol and preconditioned with $6 \mathrm{ml}$ hexane. Neutral lipids were eluted with $3 \mathrm{ml}$ of chloroform/2-propanol (2:1), free fatty acids were eluted with $3 \mathrm{ml}$ of $3 \%$ acetate in ethyl ether, and phospholipids were eluted with $3 \mathrm{ml}$ of methanol. In cases where lipids from more than $1 \mathrm{mg}$ LDL were separated, it was necessary to increase the amount of chloroform/2-propanol (2:1) used to elute the neutral lipids, otherwise some cholesteryl ester could be detected in the fatty acid fraction by TLC. The original paper (35) calls for a $2 \%$ acetic acid in ethyl ether solution to elute fatty acids, however, a $3 \%$ solution was used in these studies to protonate free fatty acids more effectively. When preparing phospholipids for tissue culture experiments, acetic acid retained in the column was removed with ethyl ether before phospholipid elution. Lipid fractions not utilized immediately were dried under nitrogen, resuspended in chloroform containing $0.01 \%$ BHT, covered with argon, and stored at $-20^{\circ} \mathrm{C}$.

Chromatography. Thin-layer chromatography of phospholipids was performed on silica gel TLC plates (60A; Whatman Inc., Clifton, NJ) 
as described (36), using a solvent mixture of chloroform/isopropanol/ triethylamine/methanol/0.25\% $\mathrm{KCl}$ (90:75:54:27:18). Lipids were visualized by spraying the developed plate with $10 \%$ sulfuric acid in ethanol and charring at $120^{\circ} \mathrm{C}$ for $15 \mathrm{~min}$. For HPLC analysis, phospholipids were resuspended in chloroform and injected onto a silica column (Spherisorb, $150 \mathrm{~mm} \times 4.6 \mathrm{~mm}, 5 \mu \mathrm{m}$; Alltech Associates, Inc.) and eluted isocratically with a mobile solvent of acetonitrile/methanol/water (79:8:13) at a flow rate of $1.0 \mathrm{ml} / \mathrm{min}$. In some cases, fractions were collected from the column under sterile conditions, dried to a residue under nitrogen at $37^{\circ} \mathrm{C}$, resuspended in tissue culture medium, and incubated with endothelial cells to test for the induction of binding activity.

Monocyte adhesion assay. Two methods were used to determine the activity of MM-LDL preparations on binding. The first assay involved measuring the increase in the binding of human monocytes to HAEC, RAEC, or porcine aortic endothelial cells. These studies were performed essentially as described previously $(15,33)$. Endothelial monolayers were treated for 4-6 $\mathrm{h}$ with MM-LDL $(125-200 \mu \mathrm{g} / \mathrm{ml})$ produced enzymatically or by incubation with cocultures for $24 \mathrm{~h}$ with or without various additives or with isolated lipids. These treatment media were removed, the cells were rinsed twice with medium, and $2.5 \times 10^{5}$ monocytes/well were added to the cells for $15 \mathrm{~min}$. Unattached cells were removed by rinsing and glutaraldehyde was added to the wells. The number of adherent monocytes was determined by visual counting. The second method involved determination of the ability of MM-LDL to inhibit LPS-induced PMN binding which we have previously documented as a characteristic action of MM-LDL (33). Data were analyzed using model 1 ANOVA.

Monocyte transmigration assay. The cocultures were treated with native LDL $(250-350 \mu \mathrm{g} / \mathrm{ml})$ in the absence or presence of various test compounds for $24 \mathrm{~h}$. The culture supernatants were subsequently transferred to untreated cocultures and were incubated for an additional $24 \mathrm{~h}$. Monocytes were labeled with the fluorescent probe $\mathrm{DiI}$ at $4^{\circ} \mathrm{C}$ for $10 \mathrm{~min}$, were centrifuged to remove the excess $\mathrm{DiI}$, and the cell pellet was resuspended in medium 199 at the desired monocyte density. At the end of the second 24-h treatment, DiI-labeled monocytes were added to cocultures at $2.5 \times 10^{5}$ cells $/ \mathrm{cm}^{2}$ and were incubated for $45-90 \mathrm{~min}$ at $37^{\circ} \mathrm{C}$. The medium containing nonadherent cells was then removed and the cell layers were washed at $37^{\circ} \mathrm{C}$ to remove the loosely adherent cells on top of the endothelial monolayer. The cocultures on membranes were fixed and then mounted, and subendothelial monocytes were enumerated under a magnification of 625 . In pilot experiments culture supernatants were screened for chemotactic activity using the monocytic cell line THP-1 which resulted in values that were in agreement with those obtained using human peripheral blood monocytes (data not shown). Data were analyzed using model 1 ANOVA.

Albumin treatment of lipoproteins. Some preparations of lipoproteins were treated with albumin in PBS at a concentration of $10 \mathrm{mg}$ albumin $/ 1 \mathrm{mg} \mathrm{LDL}$ protein as described (37) for $1 \mathrm{~h}$ at $37^{\circ} \mathrm{C}$ with gentle mixing. Separation of LDL and albumin was performed by gel filtration chromatography using an FPLC system (Pharmacia LKB Biotechnology Inc., Piscataway, NJ) equipped with two Superose 6 columns connected in series (38). Samples were eluted with a buffer containing $\mathrm{NaCl}(154 \mathrm{mM})$ and EDTA $(100 \mu \mathrm{M}), \mathrm{pH} 8.0$, at a flow rate of $0.5 \mathrm{ml} /$ $\mathrm{min}$. This method facilitated complete separation of LDL and albumin as determined by $280-\mathrm{nm}$ absorbance and SDS-polyacrylamide gel electrophoresis of eluted fractions (data not shown).

Enzyme activity assays. PAF-AH activity was determined by two different methods described previously $(39,40)$. In the method described by Steinbrecher and Pritchard, $10 \mathrm{nmol}$ of the substrate 1-palmitoyl 2-[6-(7-nitrobenzoxadiazoyl)-amino] caproyl phosphatidylcholine was incubated with LDL, HDL, plasma, or purified enzyme in 1.0 $\mathrm{ml}$ PBS. The reaction was terminated by vortexing with methanol/ chloroform. The fluorescence of the aqueous layer was measured at excitation $470 \mathrm{~nm}$ and emission $533 \mathrm{~nm}$. The mass of the substrate hydrolyzed was calculated following the method described (39). When higher sensitivity was required, $2-\left[\right.$ acetyl- $\left.{ }^{3} \mathrm{H}\right]$ PAF was used as substrate. The $\left[{ }^{3} \mathrm{H}\right]$ acetate generated after $s n-2$ hydrolysis was separated

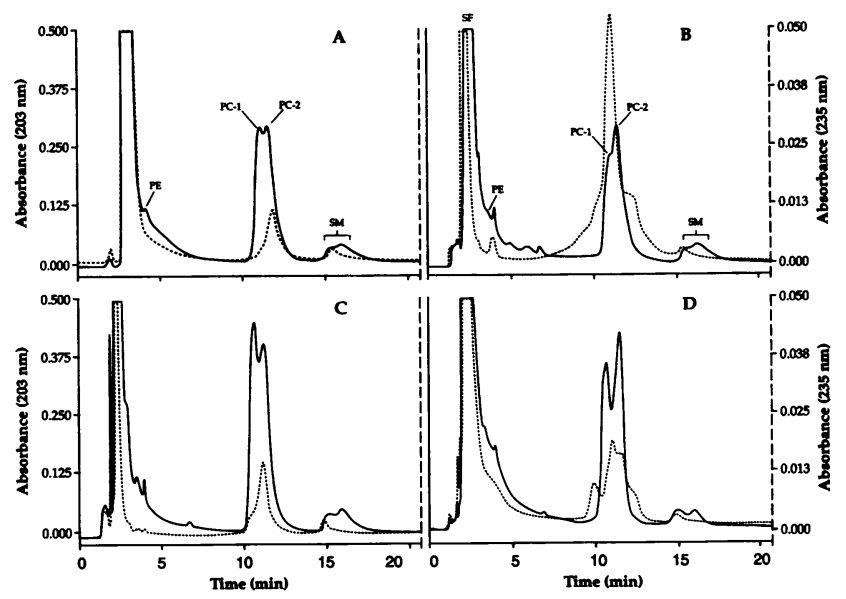

Figure 1. Analysis of MM-LDL phospholipids. HPLC analysis of phospholipids from native LDL ( $A$ and $C$ ) compared to enzymatically modified $(B)$ and coculture-modified $(D)$ MM-LDL. LDL was incubated with cocultures of human artery wall cells or soybean lipoxygenase, and linoleic acid as described in Methods. Lipids were extracted by addition of $7 \mathrm{vol}$ chloroform/methanol $(2: 1)+0.01 \%$ BHT per $1 \mathrm{vol}$ aqueous material. Total lipid extracts were separated into neutral lipids, fatty acids, and phospholipids by solid phase chromatography. Phospholipids were separated by normal phase HPLC utilizing a silica column $(4.6 \mathrm{~mm} \times 150 \mathrm{~mm}, 5 \mu \mathrm{m})$ and a solvent of acetonitrile/methanol/ water (79:8:15). Absorbance of eluting phospholipids was detected by a diode array detector (D-6000; Hitachi Instruments, Inc., San Jose,

CA). Absorbances at $203 \mathrm{~nm}(-)$ and $235 \mathrm{~nm}(--.--)$ are shown. $\mathrm{S}$, solvent front; $\mathrm{PE}$, phosphatidylethanolamine; $\mathrm{PC}$, phosphatidylcholine; and SM, sphingomyelin.

from labeled substrate by solid phase chromatography and quantitated by liquid scintillation as described previously (40). Enzymic activity is expressed in micromoles per milliliter per hour after appropriate corrections. There was complete agreement between the values obtained using the two assay methods.

Other procedures. Measurement of cell protein content and those of lipoproteins was performed by microtiter plate assay based on the method of Lowry et al. (41).

\section{Results}

HPLC analysis of MM-LDL. Phospholipids in MM-LDL produced enzymatically or by incubation with aortic cell cocultures were analyzed by HPLC (Fig. 1). MM-LDL produced enzymatically $(B)$ or by cocultures $(D)$ contained phosphatidylcholine (PC) that had less 203-nm absorbance than the corresponding native LDL ( $A$ and $C$, respectively), however, sphingomyelin typically appeared unaltered by mild oxidation. Conjugated dienes were measured by simultaneously monitoring absorbance at $235 \mathrm{~nm}$ which revealed that MM-LDL phospholipids were more oxidized than those of native LDL. A peak was detected in enzymatically modified LDL eluting near phosphatidyethanolamine that possessed strong $235 \mathrm{~nm}$ absorbance that was not detected in native LDL. Under the HPLC conditions used, PC elutes as a bimodal peak. $A$ and $C$ demonstrate the variability of the PC peaks from native LDL. However, in every experiment (12 out of 12$)$ the first peak $(P C-1)$ was diminished after treatment with oxidative enzymes $(B)$ or incubation with cocultures $(D)$. PC-1 elutes with the same retention time as a 

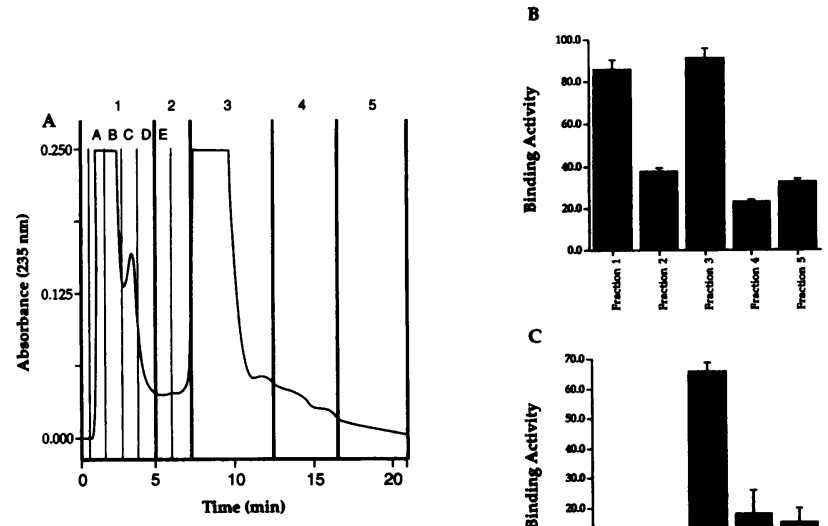

C

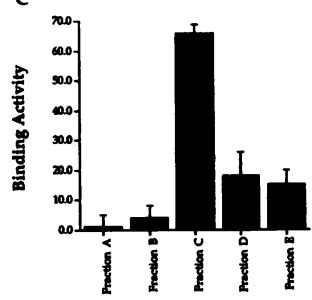

Figure 2. Effect of HPLC-isolated phospholipid fractions on binding activity. ( $A$ ) Phospholipid fractions were isolated from MM-LDL by HPLC, collected in LPS-free glass vessels, and dried to a lipid residue under a stream of nitrogen at $37^{\circ} \mathrm{C}$. Numbers $1-5$ designate the fractions tested for biological activity in $B$ and letters A-E designate the subfractions tested for biological activity in $C$. (B) Fractions 1-5 were collected as shown in $A$, suspended in DMEM (low glucose) containing 5\% FBS, and tested for induction of binding activity. $(C)$ Fractions 1-5 were collected as shown in $A$, suspended in DMEM (low glucose) containing $5 \%$ FBS, and tested for induction of binding activity. Binding activity is expressed in terms of percent inhibition of LPS-induced HL60 binding \pm SEM.

single peak produced by authentic PAPC. The second PC peak (PC-2) coelutes with 1-palmitoyl-2-linoleoyl-sn-glycero-3phosphorylcholine.

Partial characterization of active lipids in $M M-L D L$. To determine which phospholipid component(s) of MM-LDL was responsible for its biological activity, we collected five fractions ( $1-5$ ) eluting from the HPLC column (Fig. $2 A$ ). Under identical conditions, fractions were also collected from the native LDL from which the MM-LDL was produced (not shown). Each fraction was dried to a lipid residue under nitrogen, resuspended in DMEM containing 5\% FCS, and was tested for induction of endothelial binding activity. The biological activity was primarily associated with fractions 1 and 3 which included phosphatidylethanolamine and PC, respectively (Fig. $2 B$ ). Fraction 1 and a portion of fraction 2 were further separated into five subfractions $(A-E)$ (Fig. $2 A$ ) and each was tested for induction of binding activity as before (Fig. $2 C$ ). The majority of binding activity was found to be associated with subfraction C. Some activity was also associated with fractions $\mathrm{D}$ and $\mathrm{E}$, presumably due to residual active lipid eluting from the column. Fractions A and $B$, which contained the solvent front, induced no significant increase in binding activity. In separate studies we have shown that peak 1, peak 3, and material from oxidized 1-palmitoyl-2arachidonoyl-sn-glycero-3-phosphorylcholine eluting with peak 3 by HPLC cause monocyte binding (Table I). When MMLDL phospholipids were treated with dinitrophenylhydrazine, reisolated, and analyzed by HPLC, the active peak eluting near PE absorbed strongly at $360 \mathrm{~nm}$, indicating the presence of aldehydes presumably resulting from $\beta$-scission of PUFA esterified to phospholipids (data not shown). Phospholipid fractions
Table I. Effect of Oxidized Phospholipids from MM-LDL and $P A P C$ on Monocyte Binding

\begin{tabular}{lrcr}
\hline \multicolumn{1}{c}{ Additions } & Experiment 1 & $\begin{array}{r}\text { Experiment 2 } \\
\text { (MM-LDL) }\end{array}$ & $\begin{array}{r}\text { Experiment 3 } \\
\text { (Ox-PAPC) }\end{array}$ \\
\hline None & $16 \pm 4$ & $13 \pm 7$ & $27 \pm 4$ \\
MM-LDL & $44^{*} \pm 5$ & $29^{\ddagger} \pm 4$ & $41^{\ddagger} \pm 5$ \\
Phospholipids & $37^{*} \pm 4$ & $33^{*} \pm 6$ & $58^{\ddagger} \pm 6$ \\
\hline
\end{tabular}

Results from three separate experiments demonstrating the effects of MM-LDL or phospholipids produced by oxidation are shown. In the experiment shown in column 1, RAEC were untreated, treated for $4 \mathrm{~h}$ with $125 \mu \mathrm{g} / \mathrm{ml}$ of MM-LDL, or with HPLC fraction 1 phospholipids from MM-LDL. In the experiment shown in column 2, RAEC were untreated, treated for $4 \mathrm{~h}$ with $125 \mu \mathrm{g} / \mathrm{ml}$ of MM-LDL, or HPLC fraction 3 phospholipids from MM-LDL. In the expeirment shown in column 3 , endothelial cells were untreated, treated for $4 \mathrm{~h}$ with $125 \mu \mathrm{g} / \mathrm{ml}$ of MM-LDL, or an amount of oxidized PAPC equivalent to the amount of PAPC that would be found in $100 \mu \mathrm{g} / \mathrm{ml}$ of MM-LDL (roughly 10 $\mu \mathrm{g} / \mathrm{ml})$. Binding of human monocytes to the endothelial cells was then determined. Data are given as monocytes bound/field $\pm \mathrm{SD} n=6$. $^{*} P$ $<0.001 ; \quad{ }^{\ddagger} P<0.01$.

collected from native LDL produced no significant increase in endothelial binding activity (data not shown).

Effect of PAF-AH on the formation of MM-LDL by cocultures. Treatment of LDL with DFP (a serine esterase inhibitor) completely inactivated PAF-AH in LDL (from 0.95 to 0.02 nmol of 1-palmitoyl 2-[6-(7-nitrobenzoxadiazoyl)-amino]caproyl phosphatidylcholine hydrolyzed in $20 \mathrm{~min}$ by $20 \mu \mathrm{g}$ LDL). Treatment of LDL with DFP before coculture modification $(D F P \cdot L D L)$ enhanced the oxidative modification and resulted in a reproducible $(28-38 \%)$ increase in monocyte transmigration (MTM) above that produced from native LDL ( $L D L)$ (Fig. $3 \mathrm{~A}$ ). Furthermore, preincubation with purified PAF-AH $\left(2 \times 10^{-2} \mathrm{U} / \mathrm{ml}\right)$ followed by removal of unassociated PAFAH $(D F P \cdot L D L+P A F-A H)$ significantly inhibited the MTM induced by DFP $\cdot$ LDL (Fig. $3 A$ ). Treatment of cocultures with PAF-AH alone had no effect on MTM $(P A F-A H)$. In additional experiments, native HDL $(500 \mu \mathrm{g} / \mathrm{ml})$ decreased the MM-LDLinduced MTM by $79 \%(L D L+H D L)$ (Fig. $3 B$ ). HDL that had previously been treated with DFP $(D F P \cdot H D L)$, to inactivate PAF-AH, did not prevent the MM-LDL-induced MTM. Reconstitution of DFP - HDL with purified PAF-AH, followed by separation of the unassociated enzyme $(D F P \cdot H D L+P A F$ $A H$ ), rendered the DFP - HDL protective against LDL oxidation and reduced the increased MTM observed in the presence of LDL + DFP - HDL by $70 \%$ (Fig. $3 \mathrm{~B}$ ). Addition of DFP-treated purified PAF-AH was ineffective in preventing the LDL oxidation or restoring the protective effect of HDL that was previously inactivated by DFP (data not shown), indicating that protection by PAF-AH derived from its enzymatic activity.

Effect of DFP on phospholipids present in HDL after coculture incubation with $L D L$. To gain insight into the mechanism by which DFP inhibits the ability of HDL to protect against LDL modification we performed the following experiment: cocultures of artery wall cells were incubated with LDL in the presence of $\mathrm{HDL}$ or DFP-treated HDL for $24 \mathrm{~h}$ at $37^{\circ} \mathrm{C}$. As previously shown in Fig. 3, native HDL was protective against coculture-induced LDL modification and DFP·HDL was not. 


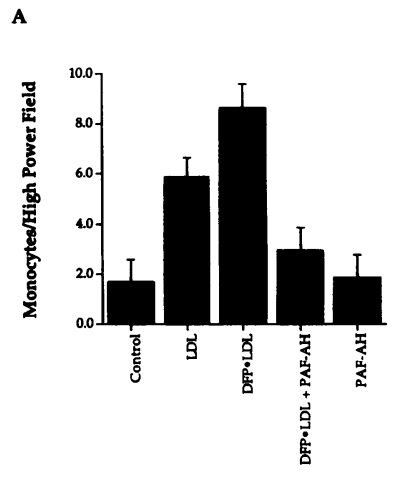

B

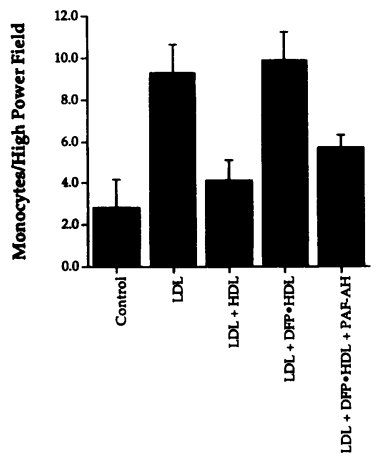

Figure 3. Effect of PAF-AH on endothelial binding activity induced by MM-LDL. $(A)$ Cocultures were incubated with native LDL $(L D L)$ or LDL that had previously been treated with 5-10 mM DFP (DFP $\cdot L D L)$ for $30 \mathrm{~min}$ at $37^{\circ} \mathrm{C}$ with gentle mixing and under argon followed by extensive dialysis to eliminate the unbound DFP. The inactivated LDL was incubated with buffer or with $2 \times 10^{-2} \mathrm{U} / \mathrm{ml}$ PAF-AH $(D F P \cdot L D L$ $+P A F-A H)$ for $2 \mathrm{~h}$ at $37^{\circ} \mathrm{C}$ with gentle mixing and under argon. The LDL preparations were subsequently dialyzed using a $100,000 \mathrm{~mol} \mathrm{wt}$ cut off membrane to eliminate any unbound PAF-AH before they were added to the appropriate cocultures. Some cocultures received LDL and purified PAF-AH. After $24 \mathrm{~h}$, conditioned medium was transferred to target cocultures and was incubated for $24 \mathrm{~h}$. Monocyte transmigration was assayed as described in Methods. $(B)$ Cocultures were incubated with $L D L$ alone $(L D L)$ or received, in addition to LDL, native HDL $(L D L+H D L)$, or native HDL that had previously been treated with 5-10 $\mathrm{mM}$ DFP for $30 \mathrm{~min}$ at $37^{\circ} \mathrm{C}$ with gentle mixing and under argon, followed by extensive dialysis to eliminate the unbound DFP. The inactivated HDL was incubated with buffer $(L D L+D F P \cdot H D L)$ or with 2 $\times 10^{-2} \mathrm{U} / \mathrm{ml}$ purified human PAF-AH $(L D L+D F P \cdot H D L+P A F-$ $A H)$ for $2 \mathrm{~h}$ at $37^{\circ} \mathrm{C}$ with gentle mixing and under argon. The HDL preparations were subsequently dialyzed using a $100,000 \mathrm{~mol}$ wt cut off membrane to eliminate any unbound PAF-AH before they were added to the appropriate cocultures. After $24 \mathrm{~h}$ of incubation, conditioned medium was transferred to target cocultures and incubated for an additional $24 \mathrm{~h}$. Monocyte transmigration was assayed as described in Methods. The values are mean $\pm \mathrm{SD}$ of the number of migrated monocytes in 20 high power fields in quadruple cocultures.

After incubation with the cocultures, LDL and HDL were separated by density gradient ultracentrifugation. Lipids were extracted from reisolated HDL and LDL and then the phospholipids were prepared for HPLC by solid phase chromatography. HPLC chromatograms of HDL from cocultures treated with LDL in presence of HDL or DFP. HDL are shown in Fig. 4. HDL reisolated from cocultures treated with $\mathrm{LDL}+$ native HDL contains a peak migrating at the position of authentic LPC, whereas HDL reisolated from cocultures treated with LDL + DFP $\cdot$ HDL contained negligible amounts of this peak. There was no LPC detected in the reisolated LDL preparations. We propose that inactivation of PAF-AH in HDL may prevent the hydrolysis of oxidized phospholipids from MM-LDL and, therefore, prevents the formation of LPC.

HPLC analysis of PAF-AH-treated MM-LDL produced by enzymatic modification. In view of the results implicating oxidized phospholipids as the active component of MM-LDL and that enzymes capable of $s n-2$ hydrolysis effectively destroy this activity, we examined the effect of PAF-AH treatment on the phospholipid profile of MM-LDL produced enzymatically.
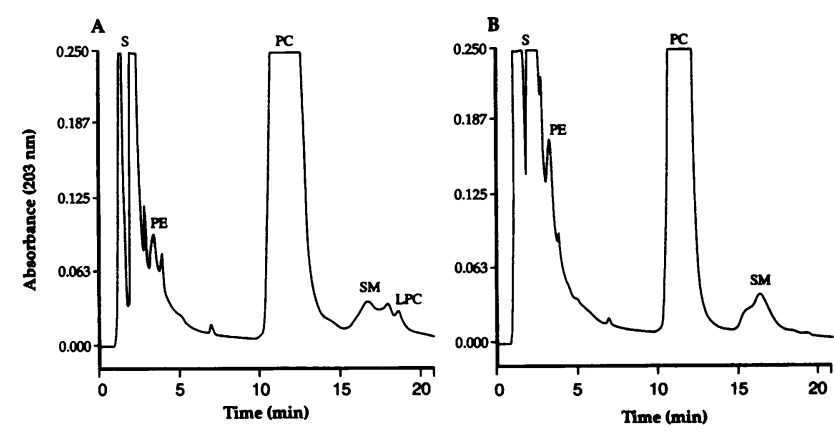

Figure 4. Effect of diisopropyl fluorophosphate on the appearance of lysophosphatidylcholine in HDL. Esterases in HDL were inactivated by incubation with $5-10 \mathrm{mM}$ DFP at $37^{\circ} \mathrm{C}$ followed by extensive dialysis. Cocultures were incubated with LDL $(350 \mu \mathrm{g} / \mathrm{ml})$ in combination with HDL $(A)$ or DFP-HDL $(B)$ from the same preparation for $24 \mathrm{~h}$ at $37^{\circ} \mathrm{C}$. LDL and HDL were reisolated from the conditioned medium by ultracentrifugation. Lipids were extracted from the HDL fraction with chloroform/methanol $(2: 1)+0.01 \%$ BHT, after which phospholipids were isolated by solid phase extraction columns. Phospholipids were separated and analyzed by HPLC as described in Fig. 1. S, solvent front; PE, phosphatidylethanolamine; PC, phosphatidylcholine; SM, sphingomyelin; and LPC, lysophosphatidylcholine.

Treatment of enzymatically oxidized MM-LDL with purified PAF-AH $\left(4 \times 10^{-2} \mathrm{U} / \mathrm{ml}\right)$ completely abolished its biological activity (Fig. $5 \mathrm{~A}$ ). Individual preparations of MM-LDL were incubated in the presence or absence of purified PAF-AH (4 $\times 10^{-2} \mathrm{U} / \mathrm{ml}$ ) overnight at $37^{\circ} \mathrm{C}$. Phospholipids were isolated as described previously and compared using HPLC and detection of oxidized phospholipids at $235 \mathrm{~nm}$. Fig. 5, $A-C$, compares the HPLC chromatograms of phospholipids before (Fig.
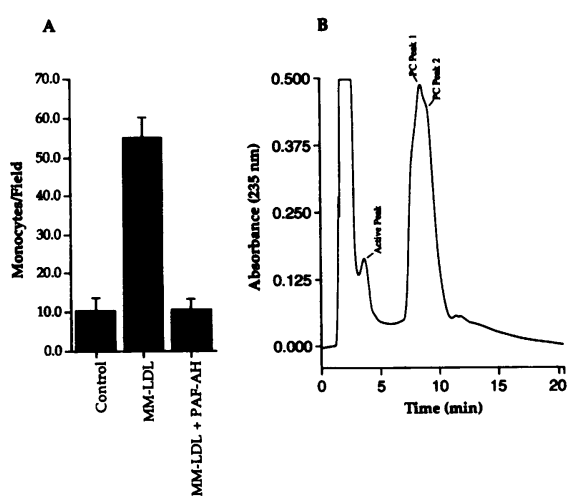

c

Figure 5. Effect of PAF-AH on the phospholipid profile and activity of MM-LDL. ( $A$ ) MM-LDL was produced by enzymatic modification with soybean lipoxygenase and linoleate as described in Methods. MM-LDL was incubated with PAF-AH $\left(4 \times 10^{-2} \mathrm{U} / \mathrm{ml}\right)$ overnight at $37^{\circ} \mathrm{C}$. HAEC were incubated with no additions (Control), MM-LDL at $125 \mu \mathrm{g} / \mathrm{ml}$ $(M M-L D L)$, or MM-LDL treated with PAF-AH $(M M-L D L+P A F-$ $A H$ ) in DMEM supplemented with $5 \% \mathrm{FBS}$ for $4 \mathrm{~h}$ at $37^{\circ} \mathrm{C}$. The medium was removed and a suspension of human monocytes was added for an additional 15-20 min. Nonadherent monocytes were removed and bound monocytes were fixed with glutaraldehyde and quantitated. Values are expressed as mean percent inhibition \pm SEM. $(B)$ and $(C)$ The phospholipids from MM-LDL $(B)$ and PAF-AH-treated MM-LDL $(C)$ were then extracted with chloroform/methanol (2:1), isolated by solid phase chromatography, and analyzed by HPLC as described in Fig. 1. 


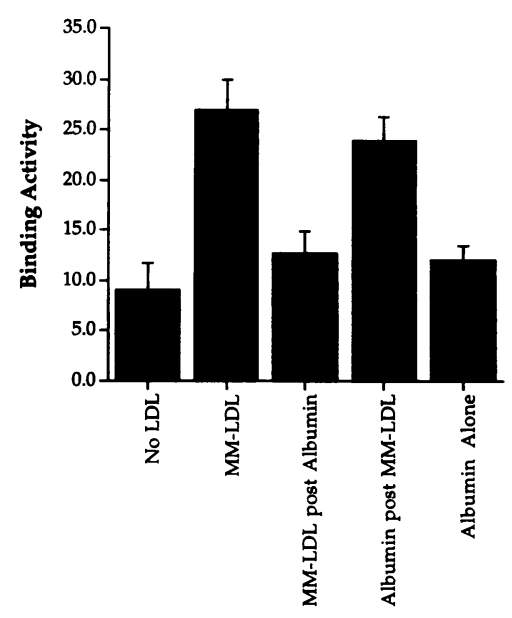

Figure 6. Transfer of active phospholipids in MM-LDL to albumin. MM-LDL was incubated with low endotoxin, essentially fatty acid free bovine serum albumin for $1 \mathrm{~h}$ at $37^{\circ} \mathrm{C}$. The albu$\mathrm{min}$ and lipoprotein were separated by density gradient ultracentrifugation. Reisolated fractions were tested for ability to induced monocyte binding to human aortic endothelial cells. MM-LDL before treatment with albumin, MM-LDL; MM-

LDL reisolated after treatment with albumin, MM-LDL post albumin; and albumin reisolated after treatment with MM-LDL, albumin post MM-LDL.

$5 B$ ) and after (Fig. $5 C$ ) treatment with PAF-AH. The peak near the solvent front that was found to be active in binding experiments (Active Peak) was reduced dramatically after PAF$\mathrm{AH}$ treatment. We also observed a moderate decrease in PC-1 (Fig. 5, $B$ and $C$ ).

Biological activity and HPLC analysis of albumin-treated $M M-L D L$. Since the phospholipid alterations in coculture-modified LDL in the region eluting near PC were similar to those in enzymatically made MM-LDL, studies were performed to determine the activity of this phospholipid and its susceptibility to PAF-AH. Other groups have shown that incubation of oxidized LDL with albumin can remove oxidized lipids from the particle (42). Because the amount of biologically active phospholipids in coculture-produced MM-LDL was a small fraction of the total phospholipids, it was necessary to enrich for oxidized phospholipids eluting near PC by incubation of MM-LDL with albumin at $10 \mathrm{mg}$ albumin/1 mg LDL protein for $1 \mathrm{~h}$ at $37^{\circ} \mathrm{C}$ with gentle rocking. Albumin and MM-LDL were reseparated by filtration and tested for induction of endothelial binding activity (Fig. 6). MM-LDL induced a threefold increase in binding activity $(M M-L D L)$. However, after reseparation, the binding activity was predominantly found in the albumin fraction (Albumin post MM-LDL). Moreover, MM-LDL reisolated after albumin treatment was ineffective at inducing endothelial cells to bind monocytes (MM-LDL post albumin). Albumin alone had no effect on binding activity. The albumin fraction containing biological activity was subjected to lipid extraction and phospholipids were purified by solid phase extraction chromatography. These polar phospholipids were resuspended in PBS and either treated with PAF-AH or buffer without enzyme overnight at $37^{\circ} \mathrm{C}$. The phospholipids were reextracted, tested for binding activity (Fig. $7 \mathrm{~A}$ ), and analyzed by HPLC (Fig. 7 $B)$. Phospholipids that were extracted from albumin that was reisolated after incubation with MM-LDL (Albumin-bound PL) were capable of inducing binding activity equal to that of the intact coculture-produced MM-LDL $(M M-L D L$, Fig. $7 A)$. However, treatment of these biologically active phospholipids with PAF-AH (Albumin-bound PL $+P A F-A H$ ) completely attenuated their ability to induce binding activity (Fig. $7 A$ ). HPLC analysis revealed that the albumin-bound phospholipids

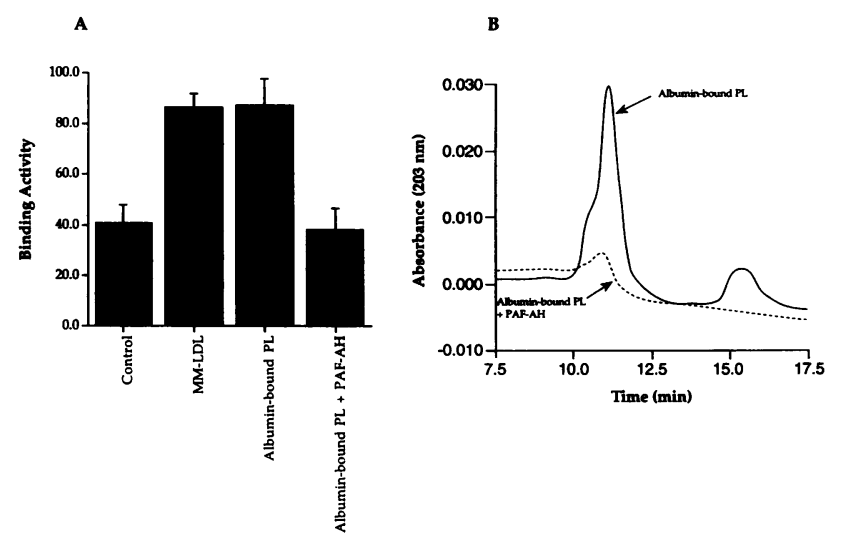

Figure 7. Effect of PAF-AH on biologocally active albumin-conjugated phospholipids. MM-LDL was incubated with albumin for $1 \mathrm{~h}$ at $37^{\circ} \mathrm{C}$ and reisolated by gel filtration chromatography as described in Methods. Lipids were extracted from the albumin fraction and the phospholipids were isolated by solid phase extraction chromatography. The phospholipids were resuspended in PBS and treated with PAF-AH $\left(4 \times 10^{-2}\right.$ $\mathrm{U} / \mathrm{ml}$ ) or buffer without enzyme overnight at $37^{\circ} \mathrm{C}$. (A) Effect of PAFAH-treated and sham-treated phospholipids on the induction of endothelial binding activity. Porcine aortic endothelial cells were incubated with no additions (Control), parent MM-LDL ( $M M-L D L)$, or phospholipid extracted from MM-LDL either treated with PAF-AH (Albumin-bound $P L+P A F-A H)$, or treated with buffer without enzyme (Albumin-bound $P L$ ) for $4 \mathrm{~h}$ at $37^{\circ} \mathrm{C}$ and tested for monocyte binding activity. (B) HPLC analysis of treated (Albumin-bound PL $+P A F-A H$, dashed line) and untreated (Albumin-bound $P L$, solid line) phospholipids reisolated after incubation with MM-LDL.

were no longer detectable by HPLC (Fig. $7 \mathrm{~B}$ ). These studies show that phospholipids produced during oxidative modification of LDL by enzymes or cocultures, induce endothelial cells to bind monocytes and suggest that PAF-AH can hydrolyze bioactive phospholipids in MM-LDL, thereby rendering them inactive.

\section{Discussion}

The mechanism(s) by which elevated plasma HDL levels are beneficial against the progression of cardiovascular and cerebrovascular disease is not well understood. Recent advances in our understanding of the atherogenicity of oxidized LDL have raised intriguing questions regarding the possible role of HDL in the prevention of LDL oxidation. To elucidate a potential mechanism by which HDL protects against the formation of MM-LDL by cocultures of human aortic wall cells, we have taken two approaches: the first was to isolate, using HPLC, particular lipid species that were responsible for the biological activity of MM-LDL produced by cocultures or enzymatic modification. The second was to determine whether PAF-AH present in HDL might decrease the content of active lipids in MMLDL, thereby destroying biological activity.

MM-LDL made by enzymatic modification or incubation with cocultures exhibited a loss of 203-nm absorbance in the first of the two PC peaks, with a concomitant increase in 235$\mathrm{nm}$ absorbance at the same retention time. Since PC containing arachidonic acid in the $s n-2$ position elutes with a retention time identical to that of PC-1 and PC containing linoleic acid elutes 
with a retention time identical to that of PC-2, we suspect that MM-LDL has undergone a moderate degree of oxidative decomposition of arachidonate-containing phosphatidylcholine and, to a lesser extent, linoleate-containing phosphatidylcholine. We also detected 235-nm absorbing material in enzymatically produced MM-LDL eluting near phosphatidyethanolamine and PC, indicating conjugated diene formation. These phospholipids were found to possess biological activity, suggesting that active phospholipids can be produced by oxidation of both phosphatidyethanolamine and PC.

Having identified the elution time and other HPLC characteristics of biologically active phospholipids we tested to see if PAF-AH altered the HPLC profile of these lipids. PAF-AH has previously been implicated in protection against $\mathrm{Cu}^{+2}$-induced oxidative modification of LDL (24). The erythrocyte PAFAH exhibits surface dilution kinetics, suggesting that hydrolysis occurs at lipid interfaces (29). It has been suggested that this enzyme acts in vivo as a scavenger of oxidatively fragmented phospholipids that are toxic to the cell (24). Red blood cells from stroke patients have lower levels of PAF-AH activity when compared with those from healthy subjects and it has been proposed that this may result in the accumulation of oxidized lipids in the cell membrane, and lead to impaired red cell deformability in patients with cerebral thrombosis (43). Since PAF is not found in red blood cells and is not an agonist for erythrocytes, it has been suggested that PAF-AH removes lipid peroxides and thus serves to maintain normal membrane deformability (43). In plasma, the majority of PAF-AH (60$70 \%$ ) is associated with LDL and the remainder with HDL. LDL was reported to have a phospholipase $A_{2}$ activity that is specific for oxidized PUFA (44) and has been suggested to be the same as PAF-AH (24), and not a fragment of apolipoprotein $B_{100}$ (45). PAF-AH in HDL was recognized by an antibody raised to PAF-AH, purified from LDL (46). This, and a series of additional experiments involving the exchange of the enzyme between LDL and HDL, established that the enzyme in the two particles is the same protein (46). It should be emphasized that the substrate specificity of PAF-AH present in LDL and HDL is quite similar to that of the erythrocyte enzyme which was used as the source of purified PAF-AH in the present studies. We observed that PAF-AH eliminated the ability of bioactive phospholipids to stimulate endothelial binding activity and that these phospholipids were no longer detectable by HPLC (Fig. 5).

Based on our observations that biologically active phospholipids in MM-LDL are substrates for PAF-AH, contain dinitrophenylhydrazine-reactive material, and can be transferred to albumin, we conclude that the active lipids in MM-LDL are most likely products produced by oxidation and fragmentation of PUFA-containing phospholipids. Work is currently under way to identify the molecular structure of these phospholipids. It has previously been shown that Ox-LDL contains cholesterolbound aldehydes, the carbonyl function of which is predominately associated with the most proximal carbon involved in a double bond (i.e., cholesteryl-arachidonate results in cholesteryl-5-oxovalerate and cholesteryl-linoleate results in cholesteryl-9-oxononanoate) (47). If this is a general phenomenon of esterified PUFA, one might expect the predominate phospholipid-bound aldehydes in LDL to be 1-acyl-2(5)-oxovaleroyl$s n$-glycero-3-phosphorylcholine and 1-acyl-2(9)-oxononanoyl$s n$-glycero-3-phosphorylcholine. It should be noted that oxi- dized phospholipids containing aldehydes up to nine carbons in the $s n-2$ position are substrates for PAF-AH (27).

In the present studies, treatment of LDL with the serine esterase inhibitor DFP resulted in a small, but significant and consistent increase in LDL-induced binding activity (Fig. $3 \mathrm{~A}$ ). We suspect that PAF-AH activity in LDL may introduce a lag phase in the production of biologically active phospholipids, during which time intrinsic PAF-AH is hydrolyzing oxidatively fragmented phospholipids. Pretreatment of LDL with DFP, thereby inactivating LDL-associated PAF-AH, renders the particles incapable of hydrolyzing fragmented phospholipids (24). The increase in biological activity of oxidatively modified DFP - LDL we observe may be attributable to an increase in the amount of biologically active phospholipids, due to the loss of the initial hydrolytic activity provided by LDL-associated PAFAH. It has previously been shown that oxidation depletes LDL of PAF hydrolytic activity $(24,48,49)$.

Inactivation and replenishment experiments imply that PAFAH plays a role in the protective effect of HDL (Fig. $3 B$ ). The role of HDL in this regard may not be to primarily serve as a source of PAF-AH, but to redistribute the hydrolase among LDL particles since not all LDL particles contain this enzyme (24). The amount of PAF-AH used in replenishment experiments was $\sim 1-2 \%$ of that found in plasma (46), however, this was sufficient for protection. The fact that $1-2 \%$ of plasma PAF-AH activity could protect against MM-LDL production suggests that the lipoproteins have considerable excess capacity for destruction of PAF-like lipids, or that added PAF-AH is more active and/or has better accessibility to target lipids than that of PAF-AH in lipoprotein particles. HDL may, alternatively, act as a sink for oxidized hydrophilic lipids that are transferred from oxidized LDL. Matsuda and colleagues (23) have demonstrated that radiolabeled LPC incorporated into $\mathrm{Ox}$ LDL spontaneously transferred into native HDL after coincubation for $30 \mathrm{~min}$ at $37^{\circ} \mathrm{C}$. Coincubation of MM-LDL and HDL, and subsequent analysis have confirmed that polar lipids readily transfer from MM-LDL to HDL (A. D. Watson and J. A. Berliner, unpublished observations). In the present study, treatment of HDL with DFP abolished the inhibitory effect of HDL against LDL modification and addition of purified erythrocyte PAF-AH restored the protective capacity of $\mathrm{HDL}$ during $\mathrm{LDL}$ oxidation (Fig. $3 B$ ). Addition of PAF-AH to LDL without inclusion of HDL in the coculture also prevented LDL modification and production of biologically active phospholipids. LPC was detected in HDL after incubation with cocultures in the presence of LDL, however, when HDL was pretreated with DFP to inactivate PAF-AH in HDL, the content of LPC in HDL was significantly less. We suggest that inactivation of PAF-AH in HDL renders the particle incapable of hydrolyzing biologically active phospholipids into LPC and fatty acid fragmentation products. Addition of DFP-treated, purified PAF-AH was ineffective in preventing the LDL oxidation or restoring the protective effect of HDL that was inactivated by DFP (data not shown). PAF itself and LPC do not appear to be the active compounds in MM-LDL since incubation of endothelial cells or cocultures with PAF or LPC in a wide range of concentrations did not induce binding activity (data not shown). We propose that HDL either: $(a)$ removes biologically active oxidized phospholipids from MM-LDL and PAF-AH in HDL subsequently hydrolyzes them into LPC and fatty acid fragmentation products; or (b) replenishes MM-LDL with active PAF-AH which then hydro- 
lyzes bioactive phospholipids within the MM-LDL particle, the products of which are transferred to HDL.

We hypothesize that in the subendothelial space, sequestered from plasma antioxidants, LDL undergoes mild lipid peroxidation. Oxidized phospholipids in MM-LDL induce inflammatory responses in endothelial cells which lead to monocyte recruitment, adhesion, and differentiation into macrophages. We further hypothesize that these active oxidized phospholipids can undergo hydrolysis by HDL-associated PAF-AH into LPC and fatty acid fragmentation products. Genetic variation of the plasma levels of $\mathrm{HDL}$ or in the content of PAF$\mathrm{AH}$ in $\mathrm{HDL}$ may regulate the content of biologically active phospholipids in the artery wall.

\section{Acknowledgments}

We thank Drs. Margaret Haberland, Jake Lusis, Laurie McLeod, Farhad Parhami, Sampath Parthasarathy, George Popják, and Brian Van Lenten for valuable discussions and suggestions; Gregory Hough for chemotaxis assays; Cynthia Harper, Darran Matthews, Thao Nguyen, Alan Wagner, and Leslie Young for excellent technical assistance; and the members of the University of California, Los Angeles, Heart Transplant Team for collecting the aortic specimens.

This work was supported by the U.S. Public Health Service grants HL-07386 (A. D. Watson), HL-45206 (A. Sevanian), HL-30568, IT 32, HL-07412, and RR 865; by the Laubisch, Rachel Israel Berro, and M.K. Grey Funds; the Cigarette and Tobacco Surtax Fund of the State of California through the Tobacco-Related Disease Research Program at the University of California (M. Navab, S.Y. Hama, and A.M. Fogelman); by the American Heart Association Minority Scientist Award (D.M. Stafforini); and by Nora Eccles Treadwell Foundation and grant IRG-178B from the American Cancer Society, and grant HL 35828 from the National Institutes of Health (D.M. Stafforini, S.M. Prescott, and T.M. McIntyre).

\section{References}

1. Steinberg, D., S. Parthasarathy, T. E. Carew, J. C. Khoo, and J. L. Witztum 1989. Beyond cholesterol. Modifications of low density lipoproteins that increase its atherogenicity. N. Engl. J. Med. 320:915-924.

2. Witztum, J. L., and D. Steinberg. 1991. Role of oxidized low density lipoprotein in atherogenesis. J. Clin. Invest. 88:1785-1792.

3. Berliner, J. A., and M. E. Haberland. 1993. Role of oxidized low density lipoprotein in atherogenesis. Curr. Opin. Lipidol. 4:373-381.

4. Esterbauer, H., J. Gebicki, H. Puhl, and G. Jürgens. 1992. The role of lipid peroxidation and antioxidants in oxidative modification of LDL. Free Radical Biol. \& Med. 13:341-390.

5. Brown, M. S., and J. L. Goldstein. 1983. Lipoprotein metabolism in the macrophage: implications for cholesterol deposition in atherosclerosis. Annu. Rev. Biochem. 52:223-261.

6. Fogelman, A. M. I. Shechter, J. Seager, M. Hokom, J. S. Child, and P. A. Edwards. 1980. Malondialdehyde alteration of low density lipoprotein leads to cholesterol ester accumulation in human monocyte-macrophage. Proc. Natl. Acad. Sci. USA. 77:2214-2218.

7. Mahley, R. W., T. L. Innerarity, K. H. Weisgraber, and S. Y. Oh. 1979. Altered metabolism (in vivo and in vitro) of plasma lipoproteins after selective chemical modification of lysine residues of the apoproteins. J. Clin. Invest. 64:743-750.

8. Morel, D. W., P. E. DiCorleto, and G. M. Chisolm. 1984. Endothelial and smooth muscle cells alter low density lipoprotein in vitro by free radical oxidation. Arteriosclerosis. 4:357-364.

9. Hoff, H. F., T. E. Whitaker, and J. O'Neil. 1992. Oxidation of low density lipoprotein leads to particle aggregation and altered macrophage recognition. $J$. Biol. Chem. 267:602-609.

10. Parthasarathy, S., D. J. Printz, D. Boyd, L. Joy, and D. Steinberg. 1986. Macrophage oxidation of low density lipoprotein generates a modified form recognized by the scavenger receptor. Arteriosclerosis. 6:505-510.

11. Henriksen, T., E. M. Mahoney, and D. Steinberg. 1983. Enhanced macrophage degradation of biologically modified low density lipoprotein. Atherosclerosis. 3:149-159.
12. Cathcart, M. K., A. K. NcNally, D. W. Morel, and G. M. Chisolm. 1989. Superoxide anion participation in human monocyte-mediated oxidation of low density lipoprotein and conversion of low density lipoprotein to a cytotoxin. $J$. Immunol. 142:1963-1969.

13. Cathcart, M. D., A. K. McNally, and G. M. Chisolm. 1991. Lipoxygenasemediated transformation of human low density lipoprotein to an oxidized and cytotoxic complex. J. Lipid Res. 32:63-70.

14. Hessler, J. R., A. L. Robertson, Jr., and G. M. Chisolm. 1979. LDLinduced cytotoxicity and its inhibition by HDL in human vascular smooth muscle and endothelial cells in culture. Atherosclerosis. 32:213-229.

15. Berliner, J. A., M. C. Territo, A. Sevanian, S. Ramin, J. A. Kim, B Bamshad, M. Esterson, and A. M. Fogelman. 1990. Minimally modified low density lipoprotein stimulates monocyte endothelial interactions. J. Clin. Invest. 85:1260-1266.

16. Navab, M., G. P. Hough, L. W. Stevenson, D. C. Drinkwater, H. Laks, and A. M. Fogelman. 1988. Monocyte migration into the subendothelial space of a coculture of adult human aortic endothelial and smooth muscle cells. J. Clin. Invest. 82:1853-1863.

17. Cushing, S. D., J. A. Berliner, A. J. Valente, M. C. Territo, M. Navab, F. Parhami, R. Gerrity, C. J. Schwartz, and A. M. Fogelman. 1990. Minimally modified low density lipoprotein induces monocyte chemotactic protein 1 in human endothelial cells and smooth muscle cells. Proc. Natl. Acad. Sci. USA 87:5134-5138.

18. Rajavashisth, T. B., A. Andalibi, M. C. Territo, J. A. Berliner, M. Navab, A. M. Fogelman, and A. J. Lusis. 1990. Induction of endothelial cell expression of granulocyte and macrophage colony-stimulating factors by modified low-density lipoproteins. Nature (Lond.). 344:254-257.

19. Navab, M., S. S. Imes, G. P. Hough, S. Y. Hama, L. A. Ross, R. W. Bork A. J. Valente, J. A. Berliner, D. C. Drinkwater, H. Laks, and A. M. Fogelman. 1991. Monocyte transmigration induced by modification of low density lipoprotein in cocultures of human aortic wall cells is due to induction of monocyte chemotactic protein 1 synthesis and is abolished by high density lipoprotein. J. Clin. Invest. 88:2039-2046.

20. Gordon, D. J., J. L. Probstfield, R. J. Garrison, J. D. Neaton, W. P. Castelli, J. D. Jacobs, Jr., S. Bangdiwala, and H. A. Tyroler. 1989. High-density lipoprotein cholesterol and cardiovascular disease. Circulation. 79:8-15.

21. Gordon, T., W. P. Castelli, M. C. Hjortland, W. B. Kannel, and T. R. Dawber. 1977. High density lipoprotein as a protective factor against coronary heart disease. Am. J. Med. 62:707-714.

22. Parthasarathy, S., J. Barnett, and L. G. Fong, 1990. High-density lipoprotein inhibits the oxidative modification of low-density lipoprotein. Biochim. Biophys. Acta. 1044:275-283.

23. Matsuda, Y., K. Hirata, N. Inoue, M. Suematsu, S. Kawashima, H. Akita and M. Yokoyama. 1993. High density lipoprotein reverses inhibitory effect of oxidized low density lipoprotein on endothelium-dependent arterial relaxation. Circ. Res. 72:1103-1109.

24. Stafforini, D. M., G. A. Zimmerman, T. M. McIntyre, and S. M. Prescott. 1993. The platelet activating factor acetylhydrolase from human plasma prevents oxidative modification of low density lipoprotein. Trans. Assoc. Amer. Phys. 105:44-63.

25. Demopoulos, C. A., R. N. Pinckard, and D. J. Hanahan. 1979. Platelet activating factor. Evidence for 1-O-alkyl-2-acetyl-sn-glyceryl-3-phosphorylcholine as the active component (a new class of lipid chemical mediators). J. Biol. Chem. 254:9355-9358.

26. Venable, M. E., G. A. Zimmerman, T. M. McIntyre, and S. M. Prescott. 1993. Platelet-activating factor: a phospholipid autocoid with diverse actions. $J$. Lipid Res. 34:691-702.

27. Stremler, K. E., D. M. Stafforini, S. M. Prescott, and T. M. McIntyre. 1991. Human plasma platelet-activating factor acetylhydrolase. Oxidatively fragmented phospholipids as substrates. J. Biol. Chem. 266:11095-11103.

28. Stremler, K. E., D. M. Stafforini, S. M. Prescott, G. A. Zimmerman, and T. M. McIntyre. 1989. An oxidized derivative of phosphatidylcholine is a substrate for the platelet-activating factor acetylhydrolase from human plasma. J. Biol. Chem. 264:5331-5334.

29. Stafforini, D. M., E. N. Rollins, S. M. Prescott, and T. M. McIntyre. 1993. The platelet-activating factor acetylhydrolase from human erythrocytes. Purification and properties. J. Biol. Chem. 268:3857-3865.

30. Rosenthal, A. M., and A. I. Gotlieb. 1990. Microvascular endothelial cells from porcine aorta. In Cell Culture Techniques in Heart and Vessel Research. H. M. Piper, editor. Springer-Verlag, Berlin. 117-129.

31. Fogelman, A. M., K. Sykes, B. J. Van Lenten, M. C. Territo, and J. A. Berliner. 1988. Modification of the Recalde method for the isolation of human monocytes. J. Lipid Res. 29:1243-1247.

32. Havel, R. J., H. A. Eder, and J. H. Bragdon. 1955. The distribution and chemical composition of ultracentrifugally separated lipoproteins of human serum. J. Clin. Invest. 43:1345-1353.

33. Parhami, F., Z. T. Fang, A. M. Fogelman, A. Andalibi, M. C. Territo, and J. A. Berliner. 1993. Minimally modified low density lipoprotein-induced 
inflammatory responses in endothelial cells are mediated by cyclic adenosine monophosphate. J. Clin. Invest. 92:471-478.

34. Bligh, E. G., and W. J. Dyer. 1959. A rapid method of total lipid extraction and purification. Can. J. Biochem. Physiol. 37:911-917.

35. Kaluzny, M. A., L. A. Duncan, M. V. Merritt, and D. E. Epps. 1985. Rapid separation of lipid classes in high yield and purity using bonded phase columns. J. Lipid Res. 26:135-140.

36. Touchstone, J. C., J. C. Chen, and K. M. Beaver. 1980. Improved separation of phospholipids in thin layer chromatography. Lipids. 15:61-62.

37. Kugiyama, K., T. Sakamoto, I. Misumi, S. Sugiyama, M. Ohgushi, H Ogawa, M. Horiguchi, and H. Yasue. 1993. Transferable lipids in oxidized lowdensity lipoprotein stimulate plasminogen activator inhibitor-1 and inhibit tissuetype plasminogen activator release from endothelial cells. Circ. Res. 73:335-343.

38. Hedrick, C. C., L. W. Castellani, C. H. Warden, D. L. Puppione, and A. J. Lusis. 1993. Influence of mouse apolipoprotein A-II on plasma lipoproteins in transgenic mice. J. Biol. Chem. 268:20676-20682.

39. Steinbrecher, U. P., and P. H. Prichard. 1989. Hydrolysis of phosphatidy choline during LDL oxidation is mediated by platelet activating factor acetylhy drolase. J. Lipid Res. 30:305-315.

40. Stafforini, D. M. T. M. McIntyre, and S. M. Prescott 1990. Platelet activating factor acetylhydrolase from human plasma. Methods Enzymol. 187:344-357.

41. Lowry, O. H., M. J. Rosebrough, A. L. Farr, and R. J. Randall. 1951. Protein measurement with the Folin Phenol reagent. J. Biol. Chem. 193:265-275.

42. Deigner, H. P., E. Friedrich, H. Sinn, and H. A. Dresel. 1992. Scavenging of lipid peroxidation products from oxidizing LDL by albumin alters the plasma halflife of a fraction of oxidized LDL particles. Free Rad. Res. Commun. 16:239-246.

43. Yoshida, H., K. Satoh, and S. Takamatsu. 1993. Platelet-activating factor acetylhydrolase in red cell membranes. Does decreased activity impair erythrocyte deformability in ischemic stroke patients? Stroke. 24:14-18.

44. Parthasarathy, S., and J. Barnett. 1990. Phospholipase $A_{2}$ activity of low density lipoprotein: evidence for the intrinsic phospholipase $A_{2}$ activity of apoprotein B-100. Proc. Natl. Acad. Sci. USA. 87:9741-9745.

45. Yamada, Y., D. M. Stafforini, T. Imaizumi, G. A. Zimmerman, T. M. McIntyre, and S. M. Prescott. 1994. Characterization of the platelet-activating factor acetylhydrolase from human plasma by heterologous expression in Xenopus laevis oocytes. Proc. Natl. Acad. Sci. USA. 91:10320-10324.

46. Stafforini, D. M. M. E. Carter, G. A. Zimmerman, T. M. McIntyre, and S. M. Prescott. 1989. Lipoproteins alter the catalytic behavior of the platelet activating factor acetylhydrolase in human plasma. Proc. Natl. Acad. Sci. USA. 86:2393-2397.

47. Kamido, H., A. Kukis, L. Marai, and J. J. Myher. 1993. Identification of core aldehydes among in vitro peroxidation of cholesteryl esters. Lipids. 28:331336.

48. Dentan, C., P. Lesnik, M. J. Chapman, and E. Ninio. 1994. PAF-acetherdegrading acetylhydrolase in plasma LDL is inactivated by copper- and cell mediated-oxidation. Atheroscler. Thromb. 14:353-360.

49. Ambrosio, G., A. Oriente, C. Napoli, G. Palumbo, P. Chiariello, G. Marone, M. Condorelli, M. Chiariello, and M. Triggiani. 1994. Oxygen radicals inhibit human plasma acetylhydrolase, the enzyme that catabolizes platelet-activating factor. J. Clin. Invest. 93:2408-2416. 